\title{
Interplay between structure and electronic properties of layered transition-metal dichalcogenides: Comparing the loss function of $1 \mathrm{~T}$ and $2 \mathrm{H}$ polymorphs
}

\author{
Pierluigi Cudazzo, ${ }^{1,2}$ Matteo Gatti, ${ }^{3,2,4}$ and Angel Rubio ${ }^{1,2,5}$ \\ ${ }^{1}$ Nano-Bio Spectroscopy Group, Departamento Física de Materiales, Universidad del País Vasco, Centro de Física de Materiales \\ CSIC-UPV/EHU-MPC and DIPC, Avenida Tolosa 72, E-20018 San Sebastián, Spain \\ ${ }^{2}$ European Theoretical Spectroscopy Facility (ETSF) \\ ${ }^{3}$ Laboratoire des Solides Irradiés, École Polytechnique, CNRS-CEA/DSM, F-91128 Palaiseau, France \\ ${ }^{4}$ Synchrotron SOLEIL, L'Orme des Merisiers, Saint-Aubin, BP 48, F-91192 Gif-sur-Yvette, France \\ ${ }^{5}$ Fritz-Haber-Institut der Max-Planck-Gesellschaft,Faradayweg 4-6, D-14195 Berlin-Dahlem, Germany \\ (Received 30 July 2014; revised manuscript received 16 October 2014; published 19 November 2014)
}

\begin{abstract}
Transition-metal dichalcogenides (TMD) share the same global layered structure, but distinct polymorphs are characterized by different local coordinations of the transition-metal atoms. Here we compared the $1 T$ and $2 \mathrm{H}$ families of metallic TMD, both in the bulk and in the two-dimensional forms. By means of first-principles time-dependent density functional calculations of the loss function, we established the direct connection between the low-energy plasmon properties and the crystal-structure symmetry. The different atomic environments affect the $d-d$ electron-hole excitations, which are prominent at low energies, resulting in distinct in-plane plasmon dispersions in the two families. Conversely, the different periodicity of the plasmon reappearance along the $c$ axis perpendicular to the layers can be used to distinguish the various crystal structures of TMD.
\end{abstract}

DOI: 10.1103/PhysRevB.90.205128

PACS number(s): 71.45.Lr, 71.45.Gm, 78.70.Ck, 73.21.Ac

\section{INTRODUCTION}

Layered materials in which quasi-two-dimensional sheets are taken together by weak van der Waals interactions are ideal to synthesize low-dimensional systems such as one-dimensional (1D) nanotubes and two-dimensional (2D) nanosheets [1-3]. Due to electron confinement, these systems display different properties with respect to their 3D counterpart, making them promising materials for both technological applications and fundamental studies. Among layered systems, the most studied one is probably graphite, in particular after the synthesis of its isolated sheets, namely, graphene [4]. However, the lack of a band gap in the electronic band structure of graphene makes its application in electronics and optoelectronics difficult $[5,6]$. This has stimulated the research into alternative classes of layered materials with more versatile electronic properties. Among them, special attention has been paid in recent years to the transition-metal dichalcogenides (TMD) [7-11].

These compounds have the formula $\mathrm{TX}_{2}$, where $\mathrm{T}$ is a transition metal and $\mathrm{X}$ is a chalcogen atom among $\mathrm{S}$, Se, and $\mathrm{Te}$. Their crystal structure derives from the stacking of $2 \mathrm{D} \mathrm{TX}_{2}$ sheets characterized by a layer of transition-metal atoms embedded between two layers of chalcogen atoms. Within a single sheet, the $\mathrm{T}$ and $\mathrm{X}$ atoms are bound covalently and form honeycomblike structures. By varying their stacking along the $c$ axis and depending on the relative alignment of the two $\mathrm{X}$ atoms within a single X-T-X sandwich, it is possible to obtain several classes of TMD. Among them, the most common ones are the $1 T$ and $2 H$ families (see Fig. 1). In this notation, the integer indicates the number of $\mathrm{TX}_{2}$ sheets in the unit cell, while $T$ and $H$ mean trigonal and hexagonal symmetry, respectively. In particular, in $1 T$ systems, the $\mathrm{T}$ atom is octahedrally coordinated by six neighboring $\mathrm{X}$ atoms ( $D_{3 d}$ point-group symmetry), while in $2 H$ systems, the coordination is trigonal prismatic $\left(D_{6 h}\right.$ point-group symmetry). Depending on the T species, TMD display metallic, semiconducting, or insulating behavior [7].
In general, systems made by transition metals belonging to the groups IV and VI of the periodic table tend to be semiconductors or insulator, while those involving the group $\mathrm{V}$ are metals [12]. This is due to the fact that in an ionic picture, the $\mathrm{TX}_{2}$ stoichiometry with $\mathrm{X}^{2-}$ requires four valence electrons from the transition metal for the bonding. Thus the synthesis of isolated sheets of TMDs allows one to obtain 2D systems characterized by both metallic and insulating behavior [13].

In the present work, we focus on the low-energy collective excitations of the metallic compounds, which have recently attracted considerable interest for the coexistence of competing electronic orders, namely, charge-density wave, including excitonic insulator instability, and superconductivity [14]. We calculate the dynamical charge-density response function of these materials by means of first-principles time-dependent density functional theory (TDDFT). We compare the loss function of $1 T$ polymorphs with that of members of the $2 \mathrm{H}$ family [15-22], both in the bulk and in the 2D form [23]. We characterize the different plasmon dispersions, highlighting the role of the intrinsic structural anisotropy and the effects of the crystal local fields that are responsible for the periodic reappearance of the spectra of the first Brillouin zone. Finally, through a detailed comparative analysis of the low-energy spectra where $d-d$ electron-hole excitations are prominent, we show how the loss function is a powerful tool to identify the fingerprints of the connection between the different crystal structures and the electronic properties of these layered materials.

\section{COMPUTATIONAL DETAILS}

In the present work, the key quantity is the loss function $L(\mathbf{q}, \omega)=-\operatorname{Im} \epsilon_{M}^{-1}(\mathbf{q}, \omega)$, where $\mathbf{q}$ is the momentum transfer, which can be measured by electron energy loss spectroscopy (EELS) or inelastic $\mathrm{x}$ ray scattering (IXS). The loss function can be written in terms of the 

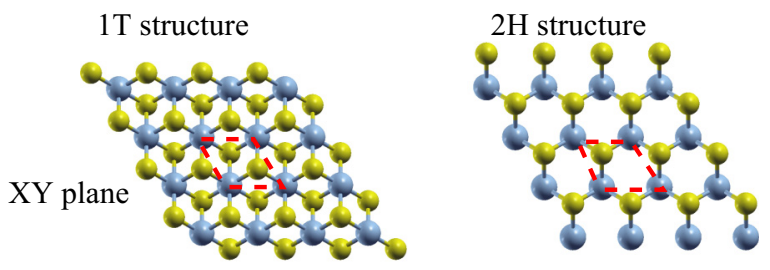

YZ plane
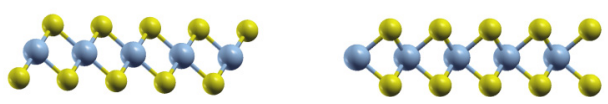

FIG. 1. (Color online) Comparison between the two-dimensional sheets of $1 T$ and $2 H$ TMD. The light-blue balls are $\mathrm{T}$ atoms and the yellow ones are $X$ atoms. The red dashed line is the projection of the unit cell in the $X Y$ plane.

real and imaginary parts of the macroscopic dielectric function $\epsilon_{M}=\epsilon_{1}+i \epsilon_{2}$ :

$$
L(\mathbf{q}, \omega)=-\operatorname{Im} \frac{1}{\epsilon_{M}(\mathbf{q}, \omega)}=\frac{\epsilon_{2}(\mathbf{q}, \omega)}{\left[\epsilon_{1}(\mathbf{q}, \omega)\right]^{2}+\left[\epsilon_{2}(\mathbf{q}, \omega)\right]^{2}} .
$$

In particular, plasmon resonances correspond to zeros of $\epsilon_{1}$ where the damping given by $\epsilon_{2}$ is not too large.

The microscopic dielectric function $\epsilon$ is related to the response function $\chi$ by [24] $\epsilon^{-1}=1+v \chi$, where $v$ is the Coulomb potential. In periodic crystals, the Fourier components of both $\chi$ and $\epsilon$ are matrices in terms of the reciprocallattice vectors $\mathbf{G}: \chi_{\mathbf{G}, \mathbf{G}^{\prime}}(\mathbf{q}, \omega)$ and $\epsilon_{\mathbf{G}, \mathbf{G}^{\prime}}(\mathbf{q}, \omega)$, where $\mathbf{q}$ belongs to the first Brillouin zone. The macroscopic dielectric function $\epsilon_{M}$ is then given by

$$
\epsilon_{M}\left(\mathbf{q}^{\prime}, \omega\right)=\frac{1}{\epsilon_{\mathbf{G G}}^{-1}(\mathbf{q}, \omega)},
$$

where $\mathbf{q}^{\prime}=\mathbf{q}+\mathbf{G}$. The difference between $\epsilon_{M}\left(\mathbf{q}^{\prime}, \omega\right)$ and $\epsilon_{\mathbf{G G}}(\mathbf{q}, \omega)$ is termed crystal local-field effects (LFE) and is related to the off-diagonal elements of the $\epsilon_{\mathbf{G G}^{\prime}}$ matrix, which are generally nonzero in inhomogeneous materials.

In the framework of time-dependent density functional theory (TDDFT) [25], the dynamical response function $\chi$ is obtained from the solution of the Dyson equation:

$$
\begin{aligned}
\chi_{\mathbf{G}, \mathbf{G}^{\prime}}(\mathbf{q}, \omega)= & \chi_{\mathbf{G}, \mathbf{G}^{\prime}}^{0}(\mathbf{q}, \omega)+\sum_{\mathbf{G}_{1}, \mathbf{G}_{2}} \chi_{\mathbf{G}, \mathbf{G}_{1}}^{0}(\mathbf{q}, \omega)\left[v_{\mathbf{G}_{1}}(\mathbf{q}) \delta_{\mathbf{G}_{1}, \mathbf{G}_{2}}\right. \\
& \left.+f_{\mathbf{G}_{1}, \mathbf{G}_{2}}^{x c}(\mathbf{q}, \omega)\right] \chi_{\mathbf{G}_{2}, \mathbf{G}^{\prime}}(\mathbf{q}, \omega),
\end{aligned}
$$

where $\chi^{0}$ is the Kohn-Sham (KS) noninteracting response function, which can be expressed in terms of KS energies and wave functions as (here $\Omega$ is the crystal volume and $f_{i}$ is the Fermi distribution)

$$
\begin{aligned}
\chi_{\mathbf{G}, \mathbf{G}^{\prime}}^{0}(\mathbf{q}, \omega)= & \frac{1}{\Omega} \sum_{\mathbf{k}, n, n^{\prime}} \frac{f_{n \mathbf{k}}-f_{n^{\prime} \mathbf{k}+\mathbf{q}}}{\omega+\varepsilon_{n \mathbf{k}}-\varepsilon_{n^{\prime} \mathbf{k}+\mathbf{q}}+i \eta}\langle n \mathbf{k}| e^{-i(\mathbf{q}+\mathbf{G}) \mathbf{r}} \\
& \times\left|n^{\prime} \mathbf{k}+\mathbf{q}\right\rangle\left\langle n^{\prime} \mathbf{k}+\mathbf{q}\left|e^{+i\left(\mathbf{q}+\mathbf{G}^{\prime}\right) \mathbf{r}^{\prime}}\right| n \mathbf{k}\right\rangle
\end{aligned}
$$

and $f^{x c}$ is the exchange-correlation kernel.

In the present work, we have considered three different prototypical TMD: $2 H-\mathrm{NbSe}_{2}, 1 T-\mathrm{VSe}_{2}$, and $1 T-\mathrm{TiTe}_{2}$. The KS eigenenergies and eigenfunctions used to determine $\chi_{0}$ have been evaluated in the local-density approximation (LDA) implemented in a plane-wave-based code [26]. In our calculations, we adopted the experimental lattice structures [27]. We used Troullier-Martins norm-conserving pseudopotentials [28] (including $3 s$ and $3 p$ semicore states in valence for $\mathrm{V}$ and $\mathrm{Ti}$ ). In the calculation of $\chi$ (Ref. [29]), we used a $24 \times 24 \times 12$ grid of $\mathbf{k}$ points and included 100 bands. The macroscopic dielectric function has been obtained by inverting a matrix of $300 \mathbf{G}$ vectors. Since in our preliminary tests we found that in the small-energy range considered here the effect of $f^{x c}$ in the adiabatic local-density approximation is negligible (see also Refs. [20] and [21]), in the following we will consider results obtained in the random-phase approximation (RPA) in which $f^{x c}=0$ and that has already been proven to be sufficient to obtain a good agreement with available experimental data $[18,19,22]$. This approximation has been used to evaluate the loss function of single-sheet TMD as well since previous works on graphene and single-wall carbon nanotubes, see e.g. [30], have demonstrated that plasmons in low-dimensional systems are well described in RPA.

\section{RESULTS AND DISCUSSION}

\section{A. In-plane loss function}

Since the different TMD polymorphs are basically distinguished by the different stacking along the $c$ axis, one would expect that the in-plane physics should be very similar for all of them. Nevertheless, we will now show how and why for the loss function in the low-energy range this is not quite the case.

In Fig. 2 we compare the low-energy ( $0-4 \mathrm{eV})$ EELS spectra of $1 T-\mathrm{VSe}_{2}$ and $1 T-\mathrm{TiTe}_{2}$ with that of $2 H-\mathrm{NbSe}_{2}$ calculated in RPA for momentum transfers $\mathbf{q}$ along $\Gamma M$, i.e., belonging to the plane of the layer. While in $2 \mathrm{H}-\mathrm{NbSe}_{2}$ (and similarly for the other metallic compounds of the $2 H$ family [19-21]) the loss function at small $\mathbf{q}$ is dominated by a single sharp peak at $\sim 1$ $\mathrm{eV}$, in the $1 T$ materials it is strongly broadened with two main features (at $\sim 0.6$ and $1.7 \mathrm{eV}$ ) and several other minor structures involving independent electron-hole pairs. Moreover, while in the $2 H$ polytypes the sharp peak has a negative dispersion as $\mathbf{q}$ increases [19-21], in $1 T-\mathrm{VSe}_{2}$ and $1 T-\mathrm{TiTe}_{2}$ the lowerand higher-energy excitations have positive and negative dispersion, respectively. In the following, we will focus on $1 T-\mathrm{VSe}_{2}$, as for $1 T-\mathrm{TiTe}_{2}$ similar considerations apply.

The main structures in the EELS spectra are plasmon resonances since they correspond to zeros of the real part of the dielectric function $\epsilon_{1}(\mathbf{q}, \omega)$ (compare Figs. 2 and 3). In both $1 T-\mathrm{VSe}_{2}$ and $2 H-\mathrm{NbSe}_{2}$, the imaginary part of the dielectric function $\epsilon_{2}(\mathbf{q}, \omega)$ is characterized by a large peak that at small $\mathbf{q}$ is located at $0.19 \mathrm{eV}$ for $2 \mathrm{H}-\mathrm{NbSe}_{2}$ and $0.24 \mathrm{eV}$ for $1 T-\mathrm{VSe}_{2}$ (see Fig. 3), below the energy of the first plasmon. These peaks of $\epsilon_{2}$ originate from the transitions involving the $d$ bands crossing the Fermi level (see Fig. 4). Thus, we can refer to the low-energy collective excitations in the EELS spectra as intraband plasmons.

While in $2 \mathrm{H}-\mathrm{NbSe}_{2}$ the higher-energy interband transitions in $\epsilon_{2}$ start at about $2 \mathrm{eV}$, in $1 T-\mathrm{VSe}_{2}$ they are much closer to the intraband plasmon. These transitions have two effects on the loss function. First, through the Kramers-Kronig relations, they cause rapid oscillations on $\epsilon_{1}$ (see Fig. 3) giving rise to the second plasmon at $1.70 \mathrm{eV}$ in $1 T-\mathrm{VSe}_{2}$, which can be 

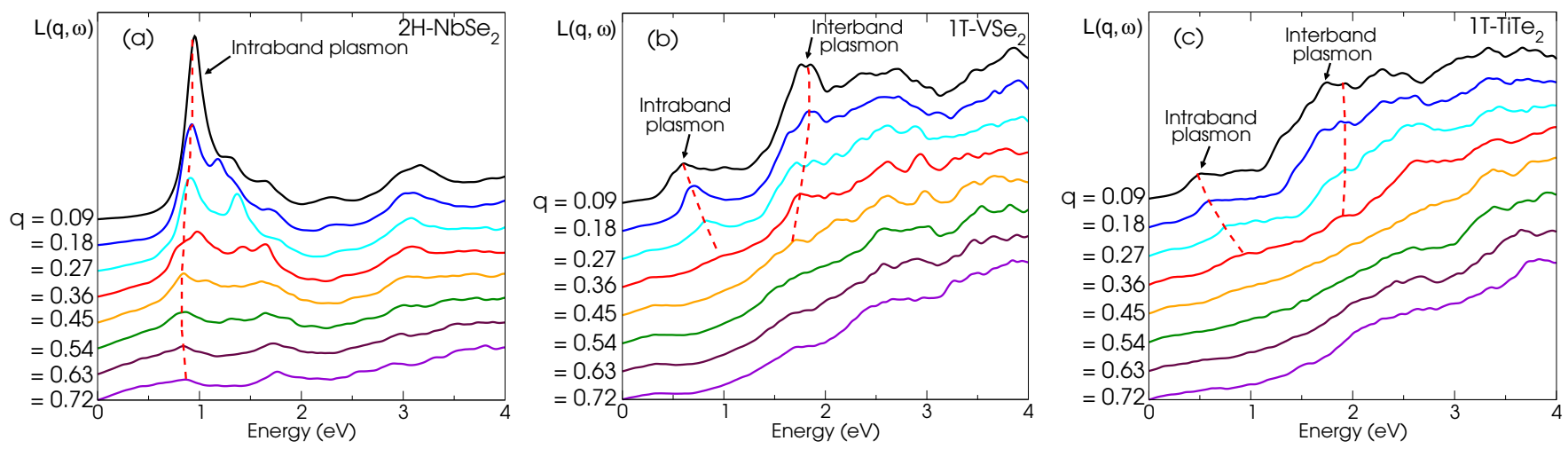

FIG. 2. (Color online) Loss functions $[L(\mathbf{q}, \omega)]$ of (a) $2 \mathrm{H}-\mathrm{NbSe}_{2}$, (b) $1 T-\mathrm{VSe}_{2}$, and (c) $1 T$ - $\mathrm{TiTe}_{2}$, calculated at the RPA level for momentum transfers $\mathbf{q}$ along $\Gamma \mathrm{M}$ (in $\AA^{-1}$ ). The red dashed line is a guide for the eye.

thus ascribed to the coherent oscillation of these interband electron-hole pairs. Second, they strongly screen the lowenergy intraband plasmon in $1 T-\mathrm{VSe}_{2}$. In fact, its energy obtained taking into account only intraband transitions (we call this the "bare plasmon") is $\sim 3.7 \mathrm{eV}$. Thus interband transitions cause a redshift of the bare plasmon by $\sim 3.1 \mathrm{eV}$. This screening effect is smaller in $2 \mathrm{H}-\mathrm{NbSe}_{2}$, where the redshift of the bare plasmon induced by the interband transitions is $\sim 2.4 \mathrm{eV}$. This happens because in this case interband transitions are weaker and occur at higher frequency. Therefore, from this analysis, we can conclude that the qualitative difference between the loss functions of $1 T$ and $2 H$ materials is due to the different energy gap in $\epsilon_{2}$ between the intraband excitations and the onset of interband transitions.

We can rationalize this finding by comparing the band structure of $1 T-\mathrm{VSe}_{2}$ and $2 \mathrm{H}-\mathrm{NbSe}_{2}$ (see Fig. 4). In both $2 \mathrm{H}$ and $1 T$ systems, for each $\mathrm{TX}_{2}$ unit, the in-plane $p$ orbitals of the $\mathrm{X}$ atoms combine with the in-plane $d$ orbitals of the $\mathrm{T}$ atom, giving rise to in-plane covalent $\sigma$ bonds. The formation of metal-chalcogen bonding and antibonding states results in a $\sigma-\sigma^{*}$ gap opening in the band structure. Since in the formation of covalent $\sigma$ bonds there is a partial charge transfer from the metal to the chalcogen atoms, the $\mathrm{T} d$ orbitals

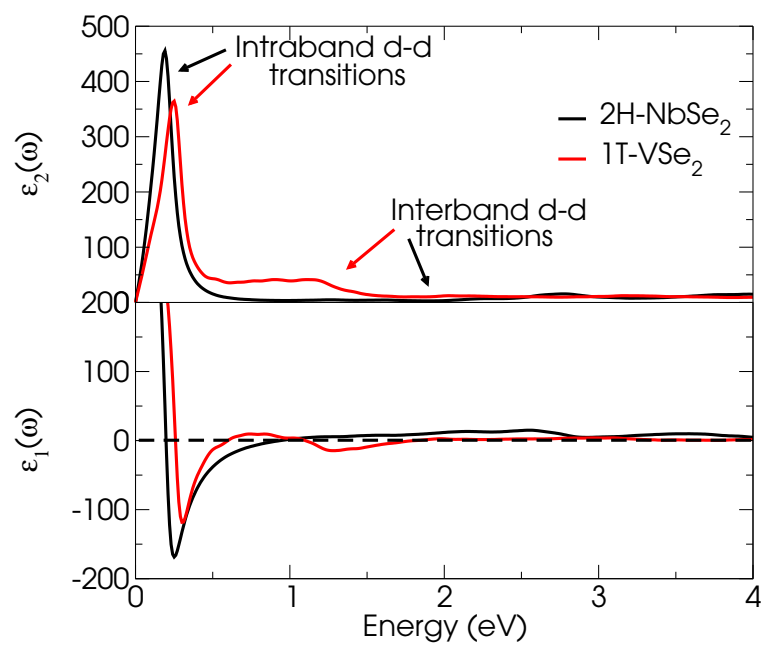

FIG. 3. (Color online) Calculated real and imaginary parts of the macroscopic dielectric function $\epsilon_{M}=\epsilon_{1}+i \epsilon_{2}$ of $2 \mathrm{H}-\mathrm{NbSe}_{2}$ and $1 T$ $\mathrm{VSe}_{2}$ for $\mathbf{q}=0.07 \AA^{-1}$ along $\Gamma \mathrm{M}$. are partially emptied and give rise to the group of $d$ bands inside the $\sigma-\sigma^{*}$ gap. These $d$ states set the metallic character of the system. Finally, the $s$ and $p_{z}$ orbitals of the $\mathrm{X}$ atoms are not involved in covalent bonds and give rise to two $s$ and two $\pi$ nonbonding states responsible for the weak van der Waals interlayer interaction. In particular, the latter are close to the Fermi level, while the former are located below the $\sigma$ bands.

The different geometrical surroundings, i.e., the crystal field, seen by the transition metal in the octahedral and trigonal prismatic structure split the $d$ manifold in a different way in $1 T$ and $2 H$ crystal structures [12], giving rise to a different
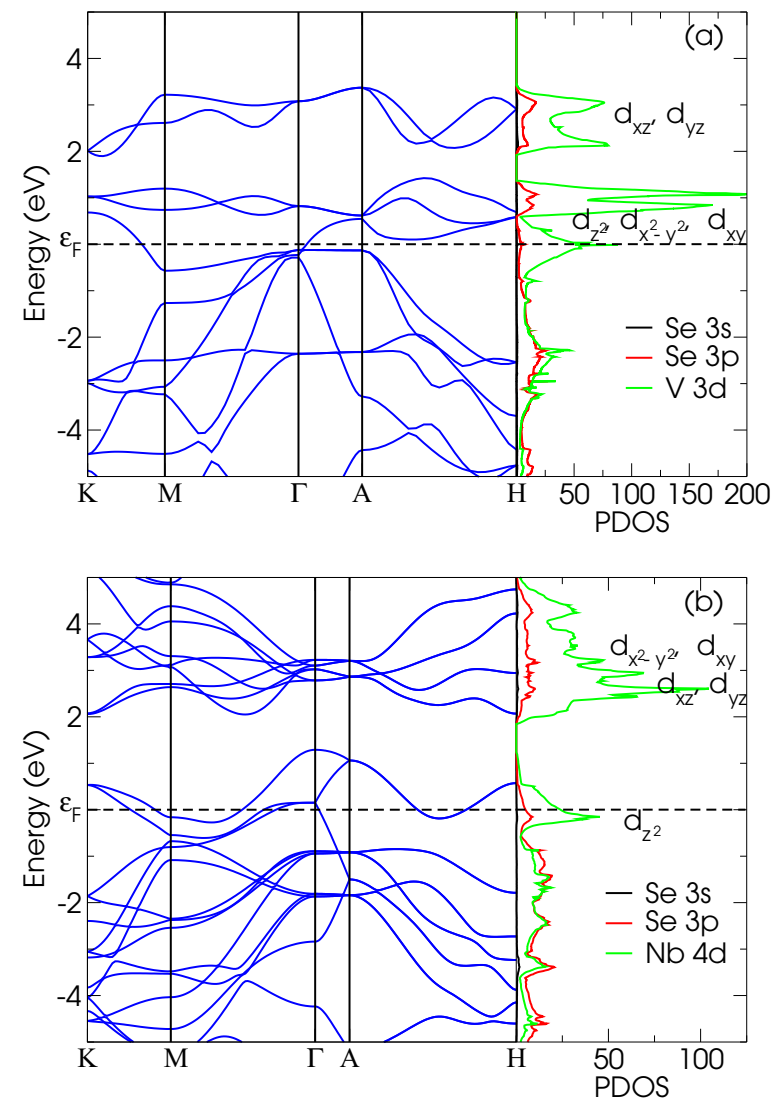

FIG. 4. (Color online) Band structure and projected density of states of (a) $1 T-\mathrm{VSe}_{2}$ and (b) $2 \mathrm{H}-\mathrm{NbSe}_{2}$. The zero of the vertical axis corresponds to the Fermi level. 


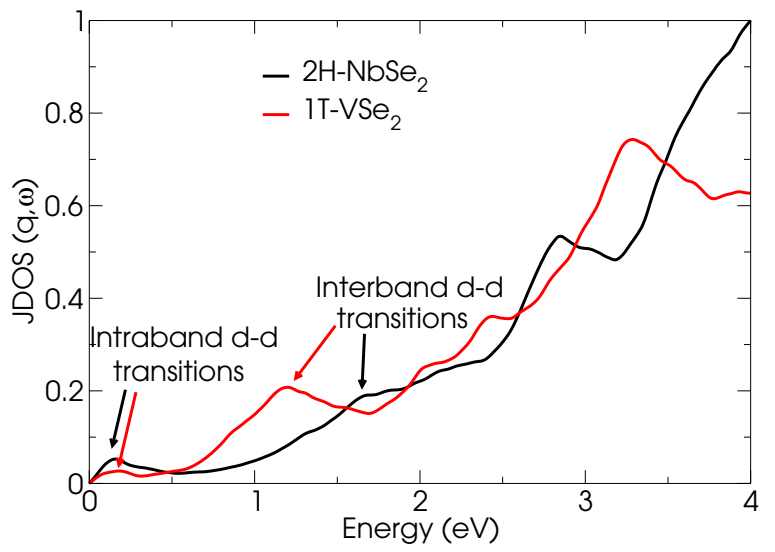

FIG. 5. (Color online) Comparison between the JDOS of $2 \mathrm{H}$ $\mathrm{NbSe}_{2}$ and $1 T-\mathrm{VSe}_{2}$ evaluated at $\mathbf{q}=0.09 \AA^{-1}$ along $\Gamma \mathrm{M}$. Both curves have been renormalized to the value of the JDOS of $2 \mathrm{H}-\mathrm{NbSe}_{2}$ at $\omega=4 \mathrm{eV}$.

behavior of the low-energy plasmon in the two materials. In both cases, the five degenerate $d$ states of the isolated atom give rise to two groups of subbands at the $\Gamma$ point (see Fig. 4). In the $1 T$ systems, having a $D_{3 d}$ point-group symmetry, the lower-energy group contains three bands that in ascending-energy order are related to $d_{z^{2}}, d_{x^{2}-y^{2}}$, and $d_{x y}$ orbitals, respectively; the higher-energy group involves two bands with $d_{x z}$ and $d_{y z}$ character. Instead, in the $2 H$ systems, having a $D_{6 h}$ point-group symmetry, the lower-energy group consists of a single band with $d_{z^{2}}$ character per layer. As a consequence, in $2 H$ compounds, the $d_{z^{2}}$ bands crossing the Fermi energy are isolated from the other unoccupied $d$ states [compare Figs. 4(a) and 4(b)].

We can evaluate the effect of the different $d$ band structures by comparing the joint density of states (JDOS) in the two materials (see Fig. 5). The first peak due to intraband transitions at $\sim 0.2 \mathrm{eV}$ is similar in both compounds. However, in the energy window between 0.5 and $1.5 \mathrm{eV}$, the JDOS in $1 T-\mathrm{VSe}_{2}$ is much larger than in $2 \mathrm{H}-\mathrm{NbSe}_{2}$. Transitions involving the $d$ states in the first group of subbands are responsible for larger JDOS in $1 T-\mathrm{VSe}_{2}$. On the contrary, their absence causes a pseudogap opening in the JDOS of $2 \mathrm{H}-\mathrm{NbSe}_{2}$. This difference in the JDOS directly translates in the different shape of $\epsilon_{2}$ (compare Figs. 3 and 5), since in the present case [20] the oscillator-strength matrix elements in $\chi^{0}$ [see Eq. (4)] and the kernel of the Dyson equation [Eq. (3)] do not alter the conclusions based on the analysis of the band structure.

From this analysis, it is therefore possible to infer a general picture of the low-energy collective excitations in these materials. In $1 T$ materials, the presence of a group of subbands close to the Fermi level gives rise to two $d$ plasmons: a low-energy intraband plasmon with $d_{z^{2}}$ character and a higherenergy plasmon related to interband transitions involving all the $d$ states. In $2 H$ materials, on the other hand, due to the different splitting of the $d$ orbitals, only the metallic states with $d_{z^{2}}$ character are able to sustain collective excitations alone. The interband transitions between $d$ states, in fact, strongly mix with the higher-energy transitions involving $\pi$ and $\sigma$ bands related to the $p_{z}$ and $p_{x y}$ orbitals of the chalcogen atoms, giving rise to the $\pi+\sigma$ plasmon located at $8 \mathrm{eV}$ [22]. This excitation (not shown) is present in $1 T$ systems as well and is located at about $6.6 \mathrm{eV}$.

The qualitative difference in the electronic band structure between $1 \mathrm{~T}$ and $2 \mathrm{H}$ systems reflects on the plasmon dispersion as well. As it has been shown in Ref. [20], due to the weak dispersion of the $d$ bands, the bare intraband plasmon of $2 \mathrm{H}$ materials has a negative dispersion that is partially attenuated by the screening effects induced by the higherenergy interband transitions. This behavior is also seen in $1 T$ systems, where the intraband bare plasmon has an intrinsic negative dispersion. However, in this case due to the proximity of the unoccupied $d$ states to the metallic $d_{z^{2}}$ band, the screening induced by interband $d-d$ transitions is strong enough to switch the slope of the plasmon dispersion to be positive. For the same reason, the intraband plasmon is strongly damped by single-particle excitations and rapidly enters the electron-hole continuum as $\mathbf{q}$ increases. On the other hand, higher-energy interband transitions above $1.70 \mathrm{eV}$ are not so efficient in screening the second $d$ plasmon that preserves its intrinsic negative dispersion.

\section{B. Monolayers}

With this picture in mind, we can now compare the loss function of monolayer $2 \mathrm{H}-\mathrm{NbSe}_{2}$ and $1 \mathrm{~T}-\mathrm{VSe}_{2}$ (Fig. 6). The qualitative difference between the spectra of $2 \mathrm{H}$ and $1 T$ systems is preserved also when the dimensionality is reduced. In fact, as for the 3D case, while the loss function of $2 \mathrm{H}-\mathrm{NbSe}_{2}$ is dominated by a single peak related to the intraband plasmon, the loss function of $1 T-\mathrm{VSe}_{2}$ is characterized by two structures. The first one at $0.7 \mathrm{eV}$ is related to $d_{z^{2}}$ plasmon, while the second one at about $2.0 \mathrm{eV}$ is the plasmon involving all the $d$ bands. However, the lack of the interlayer interaction slightly modifies the electronic band structure in $1 T$ materials, causing a reduction of the JDOS in the energy window involving the transition between the first group of $d$ subbands. As a consequence, the damping induced by interband $d-d$ transitions on the $d_{z^{2}}$ plasmon is reduced so that its strength increases, giving rise to a well-defined peak in the loss function.

Concerning the behavior of the plasmon as a function of the momentum transfer, we note that in $2 \mathrm{H}$ systems the slope of the plasmon dispersion switches to positive as the dimensionality is reduced. As has been discussed in Ref. [23], this property is related to the depolarization effect induced by crystal local fields. Finally, in the optical limit $q<0.02 \AA^{-1}$, the plasmon frequency has a $\sqrt{q}$ dispersion [31].

\section{Out-of-plane loss function}

So far, we have analyzed the main differences between the loss function of $1 T$ and $2 H$ systems for in-plane momentum transfers. In the following, to investigate how the different crystal structures are apparent also in the anisotropy of the electronic properties, we will extend our discussion to the behavior of the collective excitations for momentum transfers perpendicular to the planes of the layers.

Figure 7 shows the real and imaginary parts of the dielectric function for $2 \mathrm{H}-\mathrm{NbSe}_{2}$ and $1 \mathrm{~T}-\mathrm{VSe}_{2}$ for a small out-of-plane q. As expected, a comparison with Fig. 3 clearly points out 

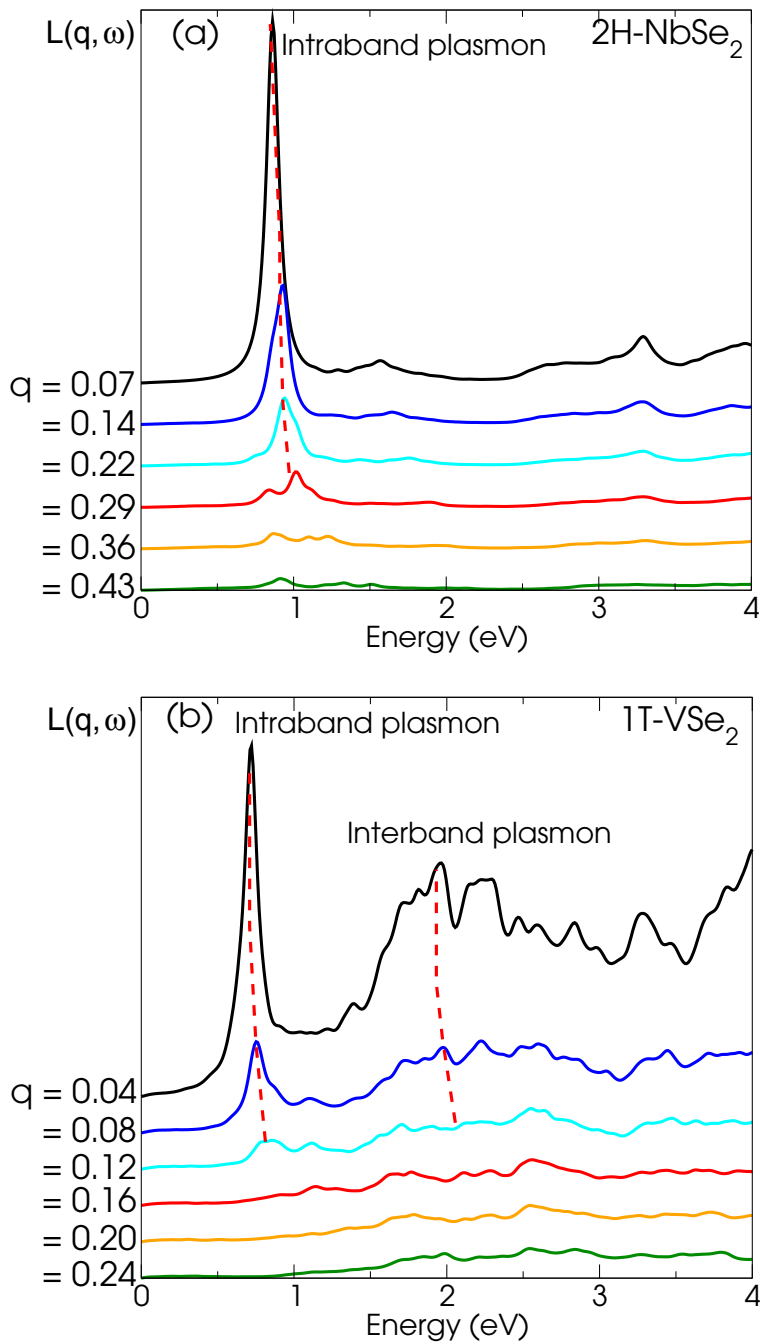

FIG. 6. (Color online) Loss functions $[L(\mathbf{q}, \omega)]$ of monolayer (a) $2 \mathrm{H}-\mathrm{NbSe}_{2}$ and (b) $1 T-\mathrm{VSe}_{2}$ calculated in RPA for momentum transfers $\mathbf{q}$ along $\Gamma \mathrm{M}$ (in $\AA^{-1}$ ). The red dashed line is a guide for the eye.

the anisotropic nature of the spectra, which is strictly related to the layered structure of these materials. Moving from the

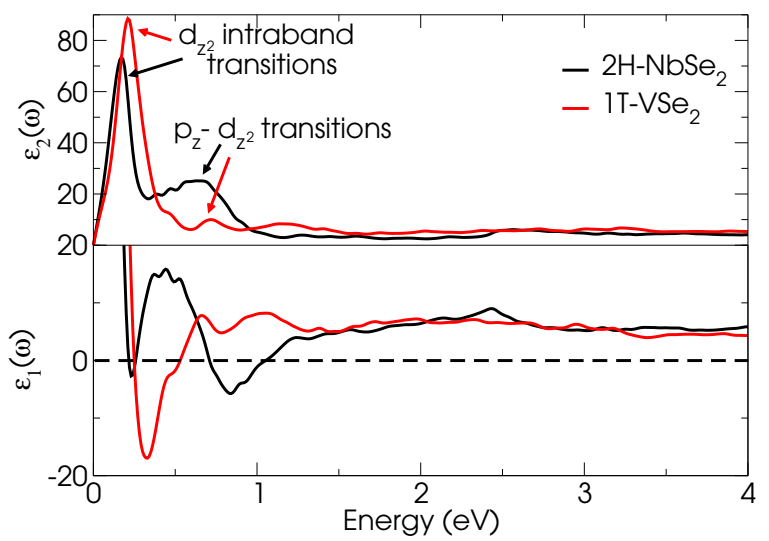

FIG. 7. (Color online) Real and imaginary parts of the macroscopic dielectric function $\epsilon_{M}=\epsilon_{1}+i \epsilon_{2}$ for $\mathbf{q}=0.17 \AA^{-1}$ along the $c$ axis for 3D stacked layers of TMD.

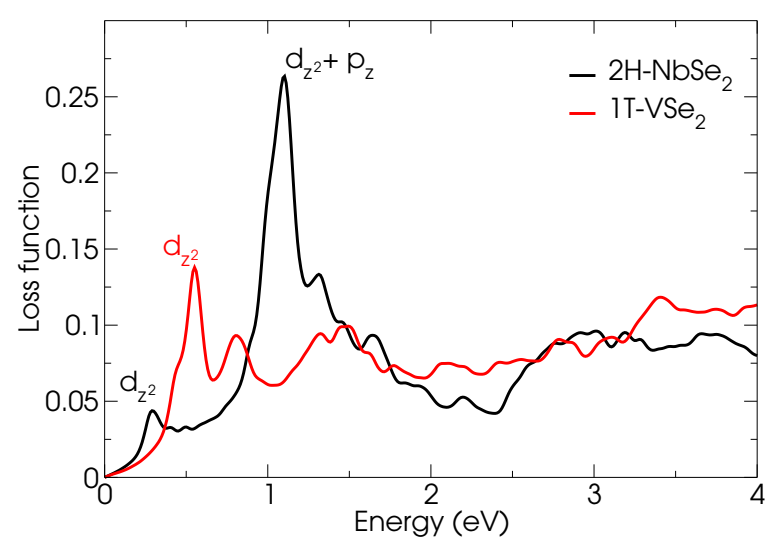

FIG. 8. (Color online) Loss function of $2 \mathrm{H}-\mathrm{NbSe}_{2}$ and $1 T-\mathrm{VSe}_{2}$ evaluated at $\mathbf{q}=0.17 \AA$ along the $z$ axis. The labels $d_{z^{2}}$ and $d_{z^{2}}+p_{z}$ indicate the character of the low- and high-energy plasmons, respectively.

in-plane to the normal direction, the strength of $\epsilon_{2}$ undergoes a global reduction in both systems. In particular, in $1 T-\mathrm{VSe}_{2}$, the interband transitions between $d$ states are completely suppressed and the out-of-plane $\epsilon_{1}$ remains positive in the region of the spectrum above $0.5 \mathrm{eV}$. As a consequence, the interband $d$ plasmon disappears (see Fig. 8).

Quite different is the behavior of the dielectric function of $2 \mathrm{H}-\mathrm{NbSe}_{2}$. In fact, in this case, in addition to the intraband peak at $0.16 \mathrm{eV}, \epsilon_{2}$ shows a higher-energy feature at $0.64 \mathrm{eV}$, which is absent for in-plane q. This new feature is related to the interband transitions between the highest $\mathrm{Se} p_{z}$ fully occupied band and the $d_{z^{2}}$ bands crossing the Fermi level. The activation of these transitions, which are dipole forbidden for in-plane momentum transfer, causes the appearance of a sharp peak in the loss function at $\sim 1.0 \mathrm{eV}$ (see Fig. 8). This feature can be interpreted as a collective excitation involving both the metallic $d_{z^{2}}$ and the $p_{z}$ bands $\left(d_{z^{2}}+p_{z}\right.$ plasmon [20]). Moreover, these $p_{z} \rightarrow d_{z^{2}}$ interband transitions strongly enhance the screening on the intraband plasmon (i.e., the lowest-energy feature in the loss function), which is redshifted by $0.3 \mathrm{eV}$ with respect to its in-plane counterpart. As in $2 H-\mathrm{NbSe}_{2}$, also in $1 T-\mathrm{VSe}_{2}$ the $p_{z} \rightarrow d_{z^{2}}$ interband transitions become visible for out-of-plane momentum transfer. However, in this case, they cannot support a collective excitation since their oscillator strength is too small.

From this analysis, we can conclude that the anisotropy acts differently in the two geometries. In $1 T$ systems, the $d$ electrons are able to support both intraband and interband collective excitations only for in-plane $\mathbf{q}$, as the interband plasmon is suppressed for out-of-plane q. In $2 H$ systems, on the other hand, intraband and interband plasmons coexist only for direction normal to the plane of the layer. For in-plane $\mathbf{q}$, only the intraband plasmon is visible in the loss function.

The different structural properties of $1 T$ and $2 \mathrm{H}$ materials have important effects on the plasmon dispersion as well, as shown in Figs. 9(a) and 9(b). In $1 T-\mathrm{VSe}_{2}$, for the absence of screening by interband $d \rightarrow d$ transitions, the intraband plasmon preserves its intrinsic negative dispersion, entering the continuum of intraband electron-hole excitations when q reaches the second Brillouin zone. In $2 \mathrm{H}-\mathrm{NbSe}_{2}$, on the 

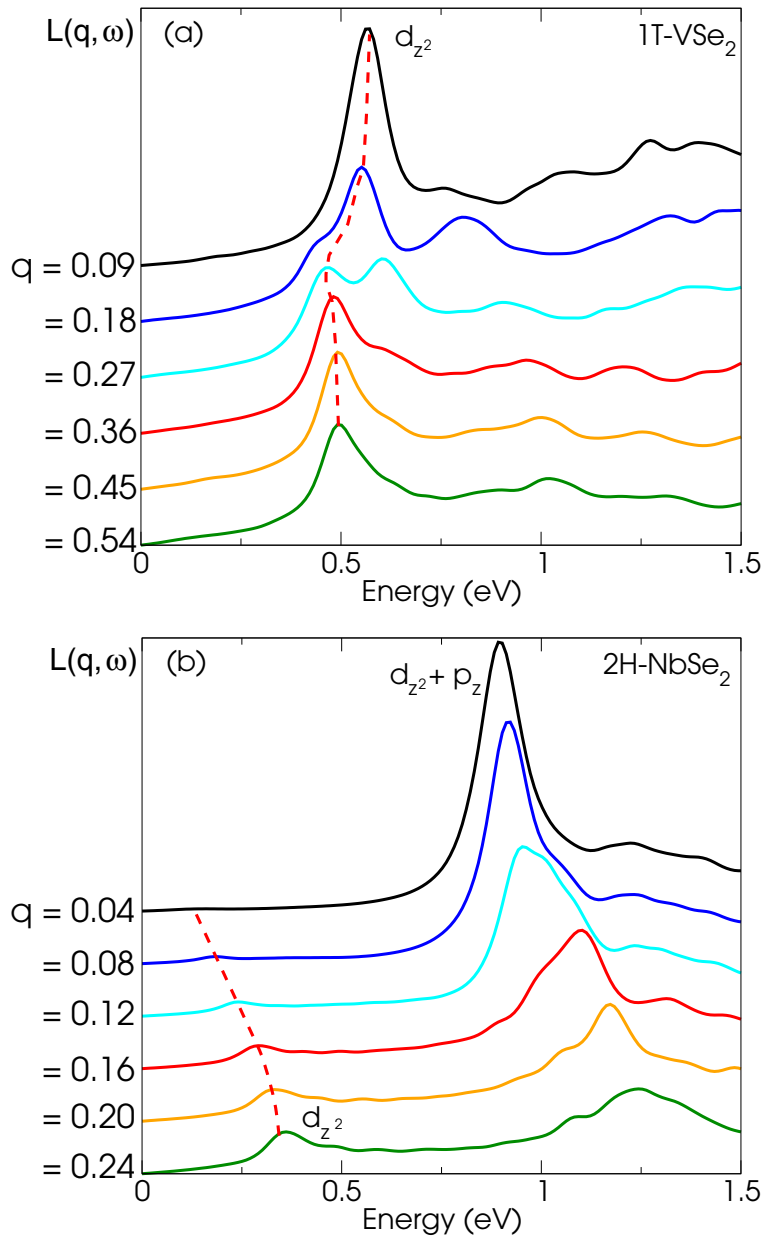

FIG. 9. (Color online) Loss functions $[L(\mathbf{q}, \omega)]$ of (a) $1 T-\mathrm{VSe}_{2}$ and (b) $2 \mathrm{H}-\mathrm{NbSe}_{2}$, calculated in the RPA for momentum transfers $\mathbf{q}$ along the $c$ axis (in $\AA^{-1}$ ). The red dashed line is a guide for the eye. The labels $d_{z^{2}}$ and $d_{z^{2}}+p_{z}$ indicate the character of the low- and high-energy plasmons, respectively.

other hand, the intraband plasmon is strongly screened by the interband $p_{z} \rightarrow d_{z^{2}}$ transitions and its intrinsic negative slope becomes positive. Interestingly, at small $\mathbf{q}$, this excitation takes a linear dispersion which is typical of an acoustic plasmon [21].

Acoustic plasmons have been theorized by Pines [32], who showed that they can occur in a two-component electron plasma consisting of "slow" and "fast" carriers. The latter can act to screen the repulsion between the former, resulting in the appearance of a plasma mode with a soundlike dispersion. This is, for instance, what has been proved in $\mathrm{MgB}_{2}$ [33], which has two bands with $\pi$ (fast carriers) and $\sigma$ (slow carriers) character that are metallic (i.e., cross the Fermi energy) or in other materials such as $\mathrm{CaC}_{6}$ [34], $\mathrm{Pb}$ [35], and $\mathrm{Pd}$ [36]. With respect to this, $2 H$-TMD are similar to $\mathrm{MgB}_{2}$ since the $d_{z^{2}}$ and $p_{z}$ bands can play the same role of the $\sigma$ and $\pi$ bands, respectively. However, at odds with $\mathrm{MgB}_{2}$, in $2 \mathrm{H}$-TMD the nonmetallic $p_{z}$ states take part in the dielectric screening through interband $p_{z} \rightarrow d_{z^{2}}$ scattering processes. As a consequence of an energy gap between $p_{z}$ and $d_{z^{2}}$ bands, these transitions induce a screening mechanism that is proper to a (gapped) insulator, and not to a metal. Since a metallic screening is a necessary condition for the energy of the screened plasmon to go to zero in the optical limit $\mathbf{q} \rightarrow 0$, we can conclude that although the $d_{z^{2}}$ collective excitation has a linear dispersion, it does not give rise to the formation of an acoustic plasmon.

On the other hand, this linear behavior is a band structure effect related to the linear dispersion of the interband $p_{z} \rightarrow d_{z^{2}}$ peak in $\epsilon_{2}$ (not shown). In fact, while the bare $d_{z^{2}}$ plasmon would have a weak negative dispersion, interband transitions completely dominate the dispersion of the screened plasmon.

Finally, we discuss the effect of crystal local fields on the plasmon dispersion. In Refs. [20,21], it was shown that similarly to other layered materials [37-40], in $2 H$ compounds LFE lead to a periodic behavior of the interband $d_{z^{2}}+p_{z}$ plasmon, which reappears at large $\mathbf{q}$ outside the first Brillouin zone. In fact, LFE induce a coupling between the plasmon at $\mathbf{q}_{r}$ in the first Brillouin zone and the independent electron-hole excitations with momentum transfer $\mathbf{q}=\mathbf{q}_{r}+\mathbf{G}_{0}$ belonging to the higher Brillouin zone identified by $\mathbf{G}_{0}$. In turn, this coupling causes the reappearance of the spectrum of the first Brillouin zone in higher zones. Moreover, it is possible to demonstrate $[20,37,38]$ that the coupling induced by LFE is zero when $\mathbf{G}_{0}$ identifies a plane in the reciprocal space where the Bragg reflection is forbidden. Due to the presence of two layers per unit cell, in $2 H$-TMD Bragg reflections along
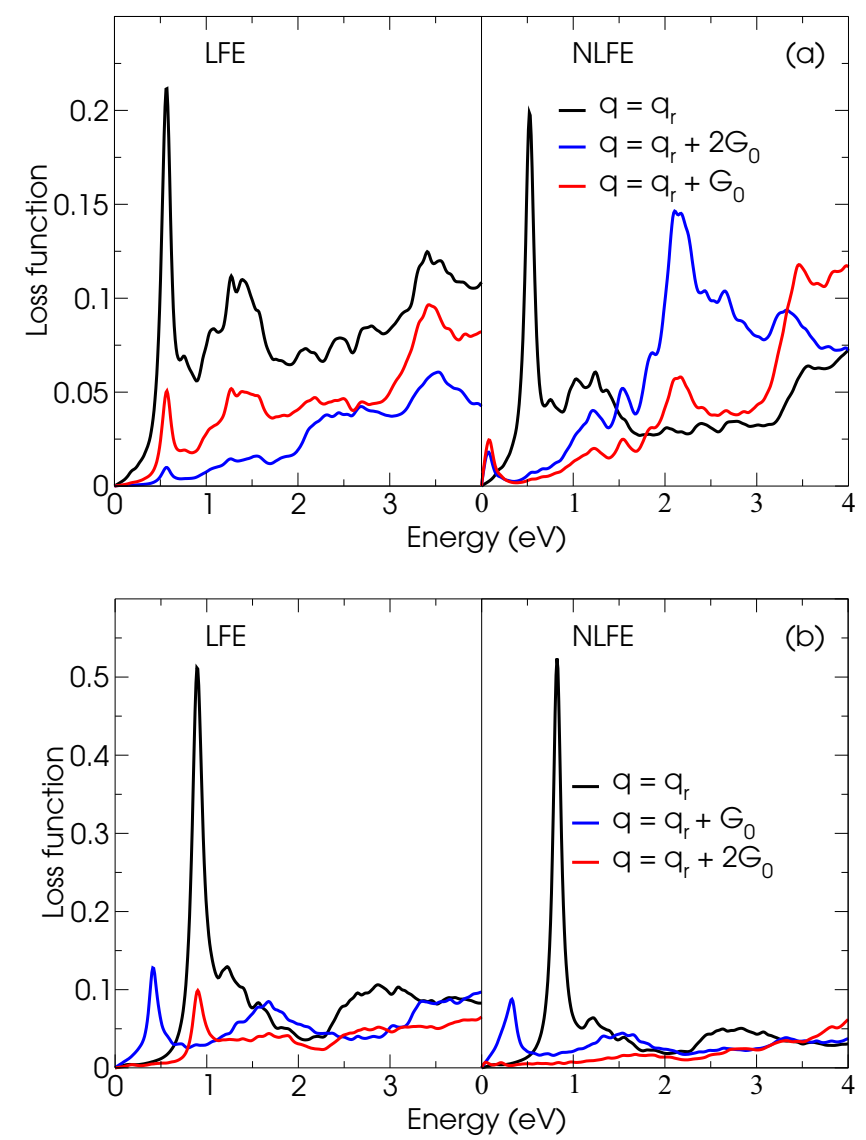

FIG. 10. (Color online) Loss function of (a) $1 T$-VSe 2 and (b) $2 H$ $\mathrm{NbSe}_{2}$ evaluated with (LFE) and without (NLFE) crystal local-field effects for different momentum transfers along the $c$ axis. Only when LFE are taken into account, for $1 T-\mathrm{VSe}_{2}$ the $d_{z^{2}}$ plasmon at $\sim 0.6 \mathrm{eV}$ $\mathbf{q}_{r}$ (black line) reappears also at $\mathbf{q}_{r}+\mathbf{G}_{0}$ (blue line) and $\mathbf{q}_{r}+2 \mathbf{G}_{0}$ (red line), while for $2 \mathrm{H}-\mathrm{NbSe}_{2}$ the $d_{z^{2}}$ plasmon at $\sim 1.0 \mathrm{eV} \mathbf{q}_{r}$ is visible at $\mathbf{q}_{r}+2 \mathbf{G}_{0}$ but not at $\mathbf{q}_{r}+\mathbf{G}_{0}$. 
the $c$ axis are allowed only when $\mathbf{G}_{0}=(0,0,2 m)$. Thus the plasmon in $2 \mathrm{H}$ compounds has a periodicity equal to two reciprocal-lattice vectors along the $c$ direction. On the other hand, 1T-TMD are characterized by a single layer per unit cell and Bragg reflections are allowed at each $\mathbf{G}_{0}=(0,0, m)$. Hence, on the basis of this analysis, in $1 T$ materials one should expect the reappearance of the intraband $d_{z^{2}}$ plasmon in each Brillouin zone. The results of the RPA calculations for $1 T-\mathrm{VSe}_{2}$ confirm these expectations [see Figs. 10(a) and 10(b). The intraband $d_{z^{2}}$ plasmon is indeed visible at $\mathbf{q}=\mathbf{q}_{r}+\mathbf{G}_{0}$, with $\mathbf{q}_{r}=0.09 \AA^{-1}$ and $\mathbf{G}_{0}=(0,0, m)$ with $m=0,1,2$, when LFE are taken into account (and is absent when LFE are neglected). Therefore, one can conclude that just by monitoring the periodicity of the low-energy plasmon, it is possible to identify the crystal structure of a given sample of layered TMD.

\section{CONCLUSIONS}

In conclusion, by comparing the loss functions and the plasmon dispersions of two different families of transition-metal dichalcogenides, we have established a detailed connection between the crystal structure and the electronic properties of these materials.

Even though all TMD share the same global crystal structure given by the stacking of 2D sheets, our first-principle calculations have proved that the different local coordination of the transition-metal atoms is directly apparent in the low-energy range of the loss spectra. In fact, the analysis of the spectra on the basis of the band structure, and its connection with the crystal-structure symmetry, has allowed us to unravel the effect of the different atomic environments. For both $2 \mathrm{H}$ and $1 T$ materials, the intraband $d_{z^{2}}$ plasmon is visible in all the directions of the momentum transfer $\mathbf{q}$, although in the $1 T$ polymorphs the in-plane $d_{z^{2}}$ plasmon energy is smaller and its dispersion is positive (in contrast to $2 \mathrm{H}$ compounds where it is negative). In the $1 T$ family, the interband $d$ plasmon is visible only for in-plane $\mathbf{q}$, while in the $2 H$ materials, the interband $d_{z^{2}}+p_{z}$ plasmon is visible only for out-of-plane $\mathbf{q}$.

Our study unambiguously shows a strong interplay between crystal structure and electronic properties in this class of materials. The high sensitivity of plasmonic properties on the local coordination of the metal atoms are an indication that structural modifications can be used, for example, to efficiently tune the dielectric properties of two-dimensional metallic TMD for plasmonic applications. Conversely, the different periodicity of the plasmon reappearance along the $c$ axis can be a useful tool to directly identify the nature of the crystal structure of these layered materials.

\section{ACKNOWLEDGMENTS}

We thank Simo Huotari for useful discussions. We acknowledge financial support from the European Research Council Advanced grant DYNamo (Grant No. ERC- 2010-AdG267374), Spanish grant (Grant No. 2010-21282-C02-01), Grupos Consolidados UPV/EHU del Gobierno Vasco (Grant No. IT578-13), and European Commission project CRONOS (Grant No. 280879-2). This research was also supported by a Marie Curie FP7 Integration Grant within the Seventh European Union Framework Programme. Computational time was granted by GENCI (Project No. 544) and BSC "Red Espanola de Supercomputacion.'
[1] H. Park, J. Park, A. K. L. Lim, E. H. Anderson, A. P. Alivisatos, and P. L. McEuen, Nature (London) 407, 57 (2000).

[2] A. I. Yanson, G. R. Bollinger, H. E. van den Brom, N. Agrait, and J. M. van Ruitenbeek, Nature 395, 783 (1998).

[3] C. Lee, Q. Y. Li, W. Kalb, X.-Z. Liu, H. Berger, R. W. Carpick, and J. Hone, Science 328, 76 (2010).

[4] K. S. Novoselov, A. K. Geim, S. V. Morozov, D. Jiang, Y. Zhang, S. V. Dubonos, I. V. Grigorieva, and A. A. Firsov, Science 306, 666 (2004).

[5] M. I. Katsnelson, Mater. Today 10, 20 (2007).

[6] A. H. Castro Neto, F. Guinea, N. M. R. Peres, K. S. Novoselov, and A. K. Geim, Rev. Mod. Phys. 81, 109 (2009).

[7] J. Wilson and A. Yoffe, Adv. Phys. 18, 193 (1969).

[8] J. N. Coleman et al. Science 331, 568 (2011).

[9] C. Ataca, H. Sahin, and S. Ciraci, J. Phys. Chem. C 116, 8983 (2012).

[10] Q. H. Wang, K. Kalantar-Zadeh, A. Kis, J. N. Coleman, and M. S. Strano, Nat. Nano 7, 699 (2012).

[11] M. Chhowalla, H. S. Shin, G. Eda, L. J. Li, K. P. Loh, and H. Zhang, Nat. Chem. 5, 263 (2013).

[12] L. F. Mattheiss, Phys. Rev. B 8, 3719 (1973).

[13] A. K. Geim and I. V. Grigorieva, Nature (London) 499, 419 (2013).

[14] K. Rossnagel, J. Phys.: Condens. Matter 23, 213001 (2011).

[15] W. Y. Liang and S. L. Cundy, Philos. Mag. 19, 1031 (1969).
[16] K. Zeppenfeld, Opt. Commun. 1, 377 (1970).

[17] G. Bell and W. Y. Liang, Adv. Phys. 25, 53 (1976).

[18] R. Schuster, R. Kraus, M. Knupfer, H. Berger, and B. Büchner, Phys. Rev. B 79, 045134 (2009).

[19] J. van Wezel, R. Schuster, A. Krönig, M. Knupfer, J. van den Brink, H. Berger, and B. Büchner, Phys. Rev. Lett. 107, 176404 (2011).

[20] P. Cudazzo, M. Gatti, and A. Rubio, Phys. Rev. B 86, 075121 (2012).

[21] M. N. Faraggi, A. Arnau, and V. M. Silkin, Phys. Rev. B 86, 035115 (2012).

[22] P. Cudazzo, K. O. Ruotsalainen, C. J. Sahle, A. Al-Zein, H. Berger, E. Navarro-Moratalla, S. Huotari, M. Gatti, and A. Rubio, Phys. Rev. B 90, 125125 (2014).

[23] P. Cudazzo, M. Gatti, and A. Rubio, New J. Phys. 15, 125005 (2013).

[24] For a review of the theoretical framework, see G. Onida, L. Reining, and A. Rubio, Rev. Mod. Phys. 74, 601 (2002).

[25] E. Runge and E. K. U. Gross, Phys. Rev. Lett. 52, 997 (1984).

[26] X. Gonze, G.-M. Rignanese, M. Verstraete, J.-M. Beuken, Y. Pouillon, R. Caracas, F. Jollet, M. Torrent, G. Zerah, M. Mikami, Ph. Ghosez, M. Veithen, J.-Y. Raty, V. Olevano, F. Bruneval, L. Reining, R. Godby, G. Onida, D. R. Hamann, and D. C. Allan, Z. Kristallogr. 220, 558 (2005). 
[27] D. E. Moncton, J. D. Axe, and F. J. Di Salvo, Phys. Rev. B 16, 801 (1977); J. Rigoult, C. Guidi-Morosini, A. Tomas, and P. Molinie, Acta Cryst. B 38, 1557 (1982); Y. Arnaud and M. Chevreton, J. Solid State Chem. 39, 230 (1981).

[28] N. Troullier and J. L. Martins, Phys. Rev. B 43, 1993 (1991).

[29] A. Marini, C. Hogan, M. Grüning, and D. Varsano, Comp. Phys. Commun. 180, 1392 (2009).

[30] C. Kramberger, R. Hambach, C. Giorgetti, M. H. Rümmeli, M. Knupfer, J. Fink, B. Büchner, L. Reining, E. Einarsson, S. Maruyama, F. Sottile, K. Hannewald, V. Olevano, A. G. Marinopoulos, and T. Pichler, Phys. Rev. Lett. 100, 196803 (2008).

[31] K. Andersen and K. S. Thygesen, Phys. Rev. B 88, 155128 (2013).

[32] D. Pines, Can. J. Phys. 34, 1379 (1956).

[33] V. M. Silkin, A. Balassis, P. M. Echenique, and E. V. Chulkov, Phys. Rev. B 80, 054521 (2009).
[34] J. P. Echeverry, E. V. Chulkov, P. M. Echenique, and V. M. Silkin, Phys. Rev. B 85, 205135 (2012).

[35] X. Zubizarreta, V. M. Silkin, and E. V. Chulkov, Phys. Rev. B 87, 115112 (2013).

[36] V. M. Silkin, I. P. Chernov, Yu. M. Koroteev, and E. V. Chulkov, Phys. Rev. B 80, 245114 (2009).

[37] R. Hambach, Ph.D. thesis, Ecole Polytechnique, 2010; http://etsf.polytechnique.fr/system/files/PhD_Hambach_2010. pdf.

[38] R. Hambach, C. Giorgetti, N. Hiraoka, Y. Q. Cai, F. Sottile, A. G. Marinopoulos, F. Bechstedt, and L. Reining, Phys. Rev. Lett. 101, 266406 (2008).

[39] Y. Q. Cai, P. C. Chow, O. D. Restrepo, Y. Takano, K. Togano, H. Kito, H. Ishii, C. C. Chen, K. S. Liang, C. T. Chen, S. Tsuda S. Shin, C. C. Kao, W. Ku, and A. G. Eguiluz, Phys. Rev. Lett. 97, 176402 (2006).

[40] K. Sturm, W. Schülke, and J. R. Schmitz, Phys. Rev. Lett. 68, 228 (1992). 